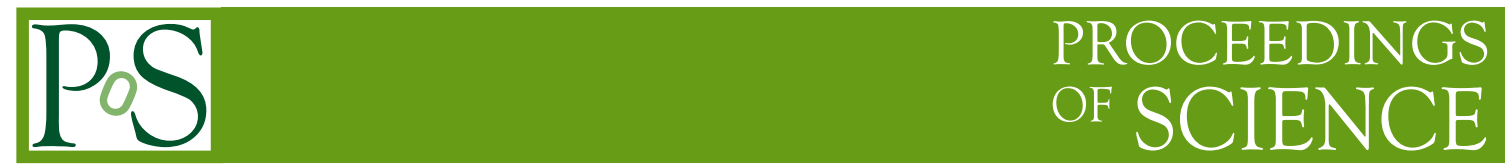

\title{
Search for Dark Matter in pp Collisions with CMS
}

\author{
Shin-Shan Yu* \\ On behalf of the CMS Collaboration \\ Department of Physics, National Central University, Chung-Li, Taiwan \\ E-mail: syu@cern.ch
}

\begin{abstract}
Searches in CMS for dark matter in final states with invisible particles recoiling against jets, top, W, Z, photon, and Higgs are presented. Various topologies are explored, covering several specific dark-matter production modes. The summary in a simplified-model framework of various searches for direct dark matter production with the CMS detector is discussed, highlighting sensitivities of the analyses under various assumptions of DM production.
\end{abstract}

38th International Conference on High Energy Physics 3-10 August 2016

Chicago, USA

${ }^{*}$ Speaker. 


\section{Introduction}

Although the dominant component of the matter in the universe is dark matter (DM), its underlying particle nature remains unknown and cannot be explained within the standard model (SM) [1]. If non-gravitational interactions exist between DM and SM particles, DM particles could be produced at the CERN LHC. The searches for dark matter at LHC, complementary to the direct detection experiments via DM-nucleon scattering and to the indirect detection experiments via DM annihilation, can probe a wide range of DM-SM interaction types. The CMS searches at Run II use the benchmark simplified models in Ref. [2] to interpret results. In these models, the minimal set of parameters include coupling structure, the mediator and DM masses $M_{\mathrm{Med}}$ and $m_{\mathrm{DM}}$, and coupling of mediator to SM and DM particles $g_{\mathrm{q}}$ and $g_{\mathrm{DM}}$. One way to observe DM particles at LHC is through their recoil off of SM particle $\mathrm{X}$ that is produced in association with the DM. The following text describes the analysis where $\mathrm{X}$ is a jet or a hadronically-decaying $\mathrm{W}$ or $\mathrm{Z}$ boson [3], a leptonically-decaying Z [4], a photon [5], a pair of top quarks $t \bar{t}$ [6], or a single top quark [7], and a Higgs boson $[8,9]$. The summary of the mono-X searches is also discussed. The mono-Higgs and mono-t $\bar{t}$ searches were performed based on $2.3 \mathrm{fb}^{-1}$ of 2015 data while the rest of the mono-X searches were performed using $12.9 \mathrm{fb}^{-1}$ of 2016 data in $\sqrt{s}=13 \mathrm{TeV}$ pp collisions collected with the CMS detector [10].

\section{Mono-jet/jets/hadronic-W,Z}

We first pre-select events containing large $E_{\mathrm{T}}^{\mathrm{miss}}\left(>200 \mathrm{GeV}\right.$ ), at least one AK4 jet ${ }^{1}$ with $p_{\mathrm{T}}>100 \mathrm{GeV}$ and veto events with well-identified electrons, muons, taus, photons, and b-jets. The minimum azimuthal angle $\Delta \phi$ between the $\vec{p}_{\mathrm{T}}^{\text {miss }}$ direction and each of the first four leading AK4 jets with $p_{\mathrm{T}}$ greater than $30 \mathrm{GeV}$ is required to be greater than 0.5 . Events are further classified into mono-V or mono-jet category. An event falls into the mono-V category if $E_{\mathrm{T}}^{\mathrm{miss}}>250 \mathrm{GeV}$, the leading AK8 jet in the event has $p_{\mathrm{T}}>250 \mathrm{GeV}$ and $|\eta|<2.4$, the jet mass after pruning within 65-105 GeV [13], and the ratio of $N$-subjettiness $\tau_{2} / \tau_{1}$ less than 0.6 [14]. The rest of the preselected events fall into the mono-jet category. Ten mutually exclusive control regions in data are used in order to get a precise estimate of the dominant $\mathrm{Z}+\mathrm{jets}$ and $\mathrm{W}+\mathrm{jets}$ backgrounds: dimuon, dielectron, single-muon, single-electron, and $\gamma+$ jets events that satisfy requirements resembling the selections imposed on the mono- $\mathrm{V}$ and mono-jet categories. The $E_{\mathrm{T}}^{\text {miss }}$ in these control regions is redefined by excluding the leptons or the photon from the $E_{\mathrm{T}}^{\text {miss }}$ calculation. The resulting hadronic recoil mimics the $E_{\mathrm{T}}^{\text {miss }}$ shape of the backgrounds in the signal region. Transfer factors that take into account the difference between the signal region and the control regions in the differential cross section, branching ratio, acceptance, reconstruction and identification efficiencies are derived from simulation; whenever possible, $p_{\mathrm{T}}$-dependent NLO QCD and NLO electroweak $K$ factors extracted from theoretical calculations are applied. The shape and the normalization of the $E_{\mathrm{T}}^{\text {miss }}$ spectra for the dominant background is determined through a maximum likelihood fit, performed simultaneously across all $E_{\mathrm{T}}^{\text {miss }}$ bins in the ten control regions and the two signal regions. Figure 1

\footnotetext{
${ }^{1}$ The clustering of jets at CMS is performed with the anti-kt algorithm [11] with a distance parameter of 0.4 and 0.8 (denoted as AK4 and AK8 jets), or with the Cambridge-Aachen algorithm [12] with a distance parameter of 1.5 (denoted as CA15 jets), respectively.
} 
shows the $E_{\mathrm{T}}^{\text {miss }}$ distributions in the mono-jet and mono-V signal regions. No significant excess is observed with respect to the SM backgrounds. Limits are computed on the DM production cross section using simplified models with spin-1 or spin- 0 mediators. Vector and axial-vector mediators with masses up to $1.95 \mathrm{TeV}$ are excluded at 95\% CL. Scalar and pseudoscalar mediators with masses up to 100 and $430 \mathrm{GeV}$, respectively, are excluded at 95\% CL. The search yields an observed (expected) upper limit of $0.44(0.56)$ at $95 \% \mathrm{CL}$ on the invisible branching fraction of the $125 \mathrm{GeV}$ Higgs boson assuming SM production cross section.

\section{Mono-leptonic-Z}

We select events with $E_{\mathrm{T}}^{\text {miss }}>100 \mathrm{GeV}$, containing a pair of electrons or muons with $p_{\mathrm{T}}^{\ell \ell}>$ $60 \mathrm{GeV}$ and reject events with extra well-identified electrons, muons, taus, b-jets, and events with more than one jet. The dominant background from the ZZ/WZ diboson production is estimated with simulated events including NNLO QCD and NLO EWK corrections. The background from $\mathrm{t} \bar{t}, \mathrm{~W}+\mathrm{jets}, \mathrm{WW}, \mathrm{tW}$, and $\mathrm{Z} \rightarrow \tau \tau$ is estimated from the $e \mu$ data with a correction factor of $0.5 \sqrt{N_{e e(\mu \mu)}^{\text {data }} / N_{\mu \mu(e e)}^{\text {data }}}$ for the $e e(\mu \mu)$ channel; the factor 0.5 corrects for the branching ratio difference while the ratio $\sqrt{N_{e e}^{\text {data }} / N_{\mu \mu}^{\text {data }}}$ accounts for the difference in the reconstruction and identification efficiency between electrons and muons. Results are interpreted with DM models with vector/axial-vector mediators and the invisible decays of the Higgs boson. Assuming the SM production rate, the observed (expected) $95 \% \mathrm{CL}$ upper limit on $\mathscr{B}(\mathrm{H} \rightarrow$ inv.) is $0.86(0.70)$.

\section{Mono-photon}

We select events containing large $E_{\mathrm{T}}^{\text {miss }}(>170 \mathrm{GeV})$, at least one photon with $p_{\mathrm{T}}>175 \mathrm{GeV}$ and $|\eta|<1.44$, and veto events with well-identified electrons and muons. The minimum $\Delta \phi$ requirement in Section 2 is also applied to reduce the QCD background. The major background from the $\mathrm{Z}(\rightarrow v v) \gamma$ and $\mathrm{W}(\rightarrow \ell v) \gamma$ processes is estimated using simulated events with NNLO QCD and NLO EWK corrections and cross-checked with control data dominated by well-reconstructed $\mathrm{Z}\left(\rightarrow \ell^{+} \ell^{-}\right) \gamma$ and $\mathrm{W}(\rightarrow \ell v) \gamma$ events. Background from jets or electrons mis-identified as photons is estimated by measuring the mis-identification rates in control samples in data. Non-collision background from beam halo and the direct interaction of particles with the ECAL photodetector are estimated by fits to distributions of the photon $\phi$ and the EM cluster seed time: beam halo events tend to produce photons with $\phi \sim 0, \pi$, while the rest of the photon events tend to be uniformly distributed in $\phi$; each process also exhibits a distinctive distribution in the EM cluster seed time and one could fit the distribution in data to the templates to extract the contribution of each component. The number of events observed in data is in good agreement with the total expected background. For the simplified DM models considered, vector/axial-vector mediator masses of up to $760 \mathrm{GeV}$ are excluded for small $m_{\mathrm{DM}}$.

\section{Mono- $t \bar{t}$}

The analysis incorporates both hadronic and semileptonic $t \bar{t}$ final states in a combined search. We require $E_{\mathrm{T}}^{\text {miss }}>200$ (160) GeV for the hadronic (semileptonic) channel. The major background 


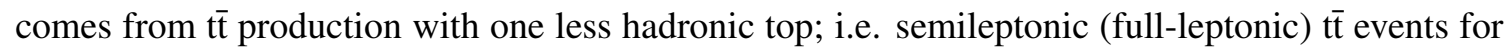
the hadronic (semileptonic) mono-tt search. A novel resolved-hadronic-top-tagging technique is developed, combining the information of the quark/gluon discriminant value for each jet, values of the b-tag discriminants, opening angles between the candidate $b$ jet and each of the jets from the candidate $\mathrm{W}$ boson, and the $\chi^{2}$ of a simultaneous kinematic fit to the top quark and $\mathrm{W}$ bosons masses using the reconstructed jet momenta, energy, and resolutions. Sensitivity is further improved by up to $30 \%$ after categorizing hadronic channel events by expected signal purity based on the number of top tags, b-tagged jets, and $\Delta \phi\left(j e t, \vec{p}_{\mathrm{T}}^{\text {miss }}\right)$. The search is interpreted in terms of DM production to place constraints on the parameter space of simplified models with spin- 0 mediators.

\section{Mono-top}

In this search we consider events with $E_{\mathrm{T}}^{\text {miss }}>250 \mathrm{GeV}$ and a hadronically-decaying top quark reconstructed using a CA15 jet. Weights calculated with the PileUp Per Particle Identification (PUPPI) algorithm [15] are applied to the particle-flow candidates to account for the impact of pileups. The CA15 jet must have $p_{\mathrm{T}}>250 \mathrm{GeV}$, a softdrop mass within $110-210 \mathrm{GeV}$ [16] and the ratio of $N$-subjettiness $\tau_{3} / \tau_{2}$ less than 0.61 . Dominant background from $\mathrm{t} \bar{t}, \mathrm{Z}+\mathrm{jets}$, and $\mathrm{W}+\mathrm{jets}$ is estimated using constraints from seven control regions: dimuon, dielectron, $\gamma+$ jets, single muon $(b-$ tagged and anti- $b$-tagged), and single electron ( $b$-tagged and anti- $b$-tagged). Similar to Section 2 , the shape and the normalization of the $E_{\mathrm{T}}^{\text {miss }}$ spectra is determined through a maximum likelihood fit, performed simultaneously across all $E_{\mathrm{T}}^{\text {miss }}$ bins in the control regions and the signal regions. Results are interpreted in terms of DM produced via a neutral flavor-changing interaction or via the decay of a colored, scalar resonance together with a single top quark. For the non-resonant model, assuming $m_{\mathrm{DM}}=10 \mathrm{GeV}$ and $a_{\mathrm{FC}}=b_{\mathrm{FC}}=0.25$, flavor-changing neutral currents of $M_{\mathrm{Med}}<$ $1.5 \mathrm{TeV}$ are excluded at 95\% CL. For the resonant model, scalar fields with $M_{\mathrm{Med}}<2.7 \mathrm{TeV}$ are excluded at $95 \% \mathrm{CL}$.

\section{Mono-Higgs}

Because Higgs boson radiation from an initial-state quark is Yukawa-suppressed and in a potential signal the Higgs boson would be part of the interaction producing the DM, mono-Higgs searches have a uniquely enhanced sensitivity to the structure of DM-SM couplings. We search for DM in the mono-Higgs channel in which the Higgs boson decays to either a pair of bottom quarks $(b \bar{b})$ or a pair of photons $(\gamma \gamma)$. The results have been interpreted using a two-Higgs-doublet model, where a vector boson $\mathrm{Z}^{\prime}$ is produced resonantly and decays into the $125 \mathrm{GeV}$ Higgs boson and an intermediate heavy pseudoscalar particle $\mathrm{A}^{0}$, which in turn decays into two DM particles. The minimum angular distance between the decay products of the Higgs boson follows the relation $\Delta R \approx 2 \times m_{\mathrm{h}} / p_{\mathrm{h}}$, where $p_{\mathrm{h}}$ is the momentum of the Higgs boson and increases with the mass of Z'. Therefore, the analysis in the bb channel is divided into two regimes: (i) a resolved regime where the Higgs boson decays to two distinctly reconstructed AK4 b jets, and (ii) a boosted regime where the Higgs boson is reconstructed by one single AK8 jet. The signal extraction is performed with a simultaneous fit to the $E_{\mathrm{T}}^{\text {miss }}$ distributions (three bins from $170-1000 \mathrm{GeV}$ for the resolved and $200-1000 \mathrm{GeV}$ for the boosted regime) in the signal and background-enriched control regions. 
The search in the $\gamma \gamma$ channel is performed by looking for an excess in the diphoton mass spectrum after requiring $E_{\mathrm{T}}^{\text {miss }}>105 \mathrm{GeV}$. Data driven techniques are used to estimate the reducible backgrounds which mainly consists of diphoton SM production. A cut-and-count based approach is used to determine the signal yield. The Z' mass range of 600 to $1863 \mathrm{GeV}$ is excluded with $95 \%$ $\mathrm{CL}$, assuming the coupling parameter $g_{\mathrm{Z}^{\prime}}=0.8$ for $\mathrm{A}^{0}$ mass at $300 \mathrm{GeV}$.

\section{Summary of dark matter searches at CMS and conclusion}

Figure 2 (left) shows the $95 \%$ CL exclusion region in the $M_{\text {Med }}-m_{\text {DM }}$ plane for di-jet searches and mono-X searches, interpreted using a DM model with a leptophobic axial vector mediator, assuming $g_{\mathrm{q}}=0.25$ and $g_{\mathrm{DM}}=1$; the dijet searches are complimentary to the mono-X searches and cover the off-shell region that mono- $\mathrm{X}$ is less sensitive to. Figure 2 (right) shows the exclusion limits for the scalar model as a function of $M_{\text {Med }}$ from various mono-X searches, assuming $g_{\mathrm{q}}=1$ and $g_{\mathrm{DM}}=1$; for smaller $M_{\text {Med }}$, the mono-tt search already has better sensitivity than the mono-jet search even with the 2015 dataset. We have performed searches for dark matter with various mono$\mathrm{X}$ final states, using $2.3 \mathrm{fb}^{-1}$ of 2015 data and $12.9 \mathrm{fb}^{-1}$ of 2016 data in $\sqrt{s}=13 \mathrm{TeV}$ pp collisions collected with the CMS detector. The data are found to be in agreement with the SM prediction. We expect updates with the full 2016 dataset in the near future.

\section{References}

[1] G. Bertone, D. Hooper, and J. Silk, "Particle dark matter: Evidence, candidates and constraints," Phys. Rept. 405, 279 (2005).

[2] D. Abercrombie et al., "Dark Matter Benchmark Models for Early LHC Run-2 Searches: Report of the ATLAS/CMS Dark Matter Forum,” arXiv:1507.00966.

[3] CMS Collaboration, "Search for dark matter in final states with an energetic jet, or a hadronically decaying $\mathrm{W}$ or $\mathrm{Z}$ boson using $12.9 \mathrm{fb}^{-1}$ of data at $\sqrt{s}=13 \mathrm{TeV}$," CMS-PAS-EXO-16-037.

[4] CMS Collaboration, "Search for dark matter in $\mathrm{Z}+E_{\mathrm{T}}^{\mathrm{miss}}$ events using $12.9 \mathrm{fb}^{-1}$ of 2016 data," CMS-PAS-EXO-16-038.

[5] CMS Collaboration, "Search for dark matter and graviton produced in association with a photon in pp collisions at $\sqrt{s}=13 \mathrm{TeV}$ with an integrated luminosity of $12.9 \mathrm{fb}^{-1}$," CMS-PAS-EXO-16-039.

[6] CMS Collaboration, "Search for dark matter in association with a top quark pair at $\sqrt{s}=13 \mathrm{TeV}$," CMS-PAS-EXO-16-005.

[7] CMS Collaboration, "Search for new physics in a boosted hadronic monotop final state using 12.9 $\mathrm{fb}^{-1}$ of $\sqrt{s}=13 \mathrm{TeV}$ data," CMS-PAS-EXO-16-040.

[8] CMS Collaboration, "Search for Dark Matter Produced in Association with a Higgs Boson Decaying to Two Photons," CMS-PAS-EXO-16-011.

[9] CMS Collaboration, "Search for dark matter in association with a Higgs boson decaying into a pair of bottom quarks at $\sqrt{s}=13 \mathrm{TeV}$ with the CMS detector," CMS-PAS-EXO-16-012.

[10] CMS Collaboration, "The CMS experiment at the CERN LHC," JINST 3, S08004 (2008).

[11] M. Cacciari, G. P. Salam, and G. Soyez, “The Anti-k(t) jet clustering algorithm,” JHEP 0804, 063 (2008). 

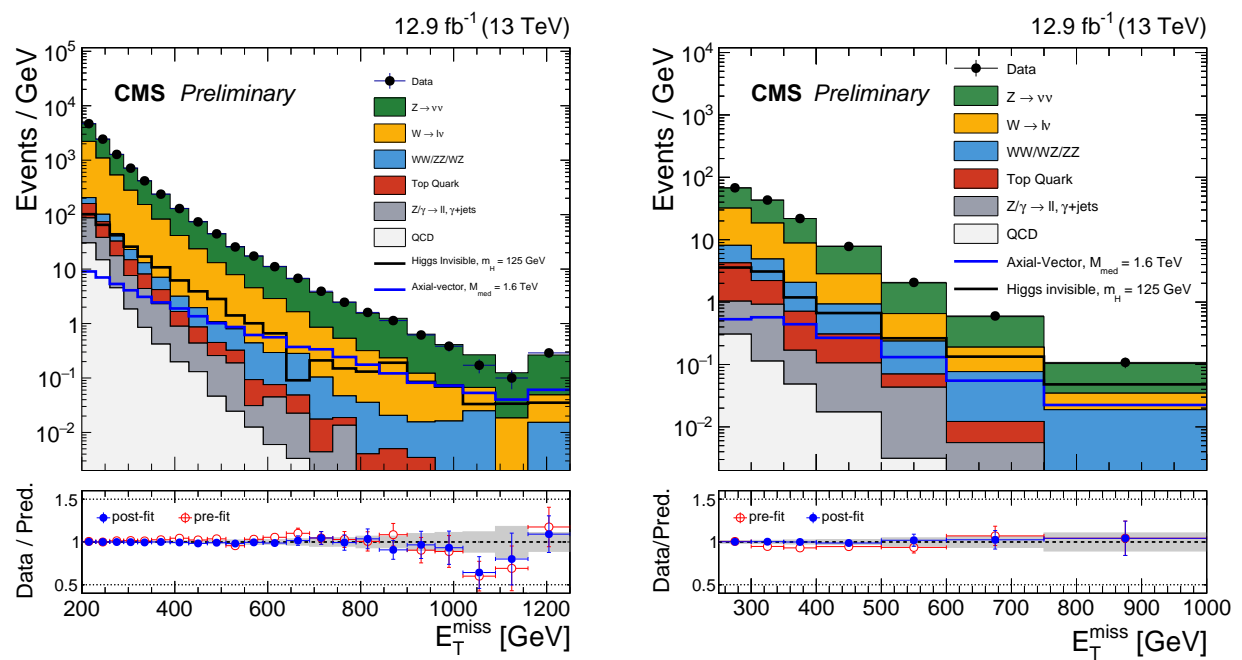

Figure 1: Observed $E_{\mathrm{T}}^{\text {miss }}$ distribution in the monojet (left) and mono-V (right) signal regions compared with the post-fit background expectations for various SM processes.
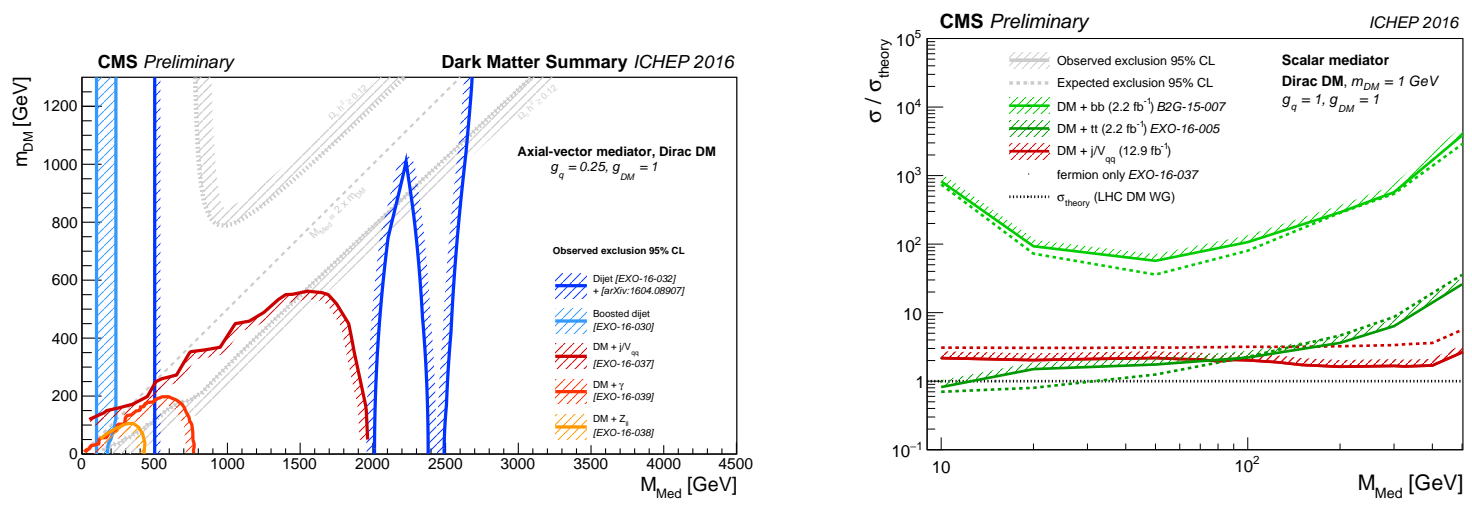

Figure 2: Left: The 95\% CL exclusion regions in $M_{\mathrm{Med}}-m_{\mathrm{DM}}$ plane for di-jet searches and various $E_{\mathrm{T}}^{\text {miss }}$ based DM searches from CMS in the leptophobic axial vector model. Right: The 95\% CL observed (fullline) and expected (dashed-line) exclusion limits for the scalar model as a function of $M_{\text {Med }}$ for different $E_{\mathrm{T}}^{\text {miss }}$ based DM searches from CMS.

[12] S. Catani, Y. L. Dokshitzer, M. H. Seymour, and B. R. Webber, "Longitudinally invariant $K_{t}$ clustering algorithms for hadron hadron collisions," Nucl. Phys. B 406, 187 (1993).

[13] S. D. Ellis, C. K. Vermilion, and J. R. Walsh, "Recombination Algorithms and Jet Substructure: Pruning as a Tool for Heavy Particle Searches," Phys. Rev. D 81, 094023 (2010).

[14] J. Thaler and K. Van Tilburg, "Identifying Boosted Objects with N-subjettiness," JHEP 1103, 015 (2011).

[15] D. Bertolini, P. Harris, M. Low, and N. Tran, “Pileup Per Particle Identification,” JHEP 1410, 059 (2014).

[16] A. J. Larkoski, S. Marzani, G. Soyez, and J. Thaler, “Soft Drop,” JHEP 1405, 146 (2014). 